\title{
ENZYMATIC BIODIESEL SYNTHESIS FROM ACID OIL USING A LIPASE MIXTURE
}

\author{
Kelly C. N. R. Pedro ${ }^{a}$, Juliana M. Parreira ${ }^{a}$, Igor N. Correia ${ }^{a}$, Cristiane A. Henriques ${ }^{a, *}$ and Marta A. P. Langone ${ }^{a, b}$ \\ aDepartamento de Química Analítica, Instituto de Química, Universidade do Estado do Rio de Janeiro, Rua São Francisco Xavier, \\ 524, 20550-900, Rio de Janeiro - RJ, Brasil \\ 'Instituto Federal de Educação, Ciência e Tecnologia, Rua Senador Furtado, 121, 20260-100 Rio de Janeiro - RJ, Brasil
}

Recebido em 09/08/2017; aceito em 04/12/2017; publicado na web em 21/12/2017

\begin{abstract}
The conventional biodiesel production process has some disadvantages. It is necessary to use refined vegetable oils with low free fatty acids (FFAs) content. An alternative route is to use low-cost acid oils in an enzymatic process. The use of lipases allows simultaneous esterification of FFAs and transesterification of triglycerides present in raw material forming alkyl esters. The aim of this work was to study the production of biodiesel using soybean oils with different acid contents (Acid Value of 8.5, 50, 90) and ethanol catalyzed by commercial immobilized lipases (Novozym 435, Lipozyme RM IM and Lipozyme TL IM). A significant decrease of acid value was observed mainly with Novozym 435 and Lipozyme RM IM. The use of a mixture of two immobilized lipases was also investigated to decrease catalyst cost and increase the amount of ester produced. The three commercial immobilized lipases were mixed in a dual system and tested for biodiesel synthesis from acid oil (AV of 8.5, 50 and 90). A positive synergistic effect occurred mainly for Lipozyme TL IM (1,3-specific lipase) and Novozym 435 (non-specific lipase) blend. The ester content doubled when this lipase mixture was used in ethanolysis of acid oil with AV of 90.
\end{abstract}

Keywords: lipase mixture; acid oil; biodiesel; soybean oil; transesterification.

\section{INTRODUCTION}

Biodiesel is a mixture of fatty acid alkyl esters that is nontoxic, biodegradable and an excellent substitute for diesel fuel. The conventional process for biodiesel production is the transesterification of refined vegetable oils with short carbon chain alcohols (methanol or ethanol) in the presence of homogeneous basic catalysts, such as sodium or potassium hydroxides, carbonates or alkoxides. This reaction produces fatty acid alkyl esters as its main product and glycerol as co-product. However, these catalytic systems have some technological problems: the recovery and purification of glycerol, the difficulty to remove basic catalysts and water from biodiesel, the production of a large amount of wastewater, and the emulsification. Moreover, they require the use of refined vegetable oils that have low free fatty acids (lower than $0.5 \mathrm{wt} . \%$ ) and water content. ${ }^{1}$ Hence, the high cost of these raw material accounts for $60-80 \%$ of the total biodiesel production cost. ${ }^{2}$

The use of non-edible oils, waste oils (waste cooking oil) and animal fats have been investigated considering the economic feasibility of the process. However, the low-cost feedstock has some disadvantages due to the high content of free fatty acid (FFA). FFAs are products from the hydrolysis of triglycerides, and they interfere with the usual alkaline-catalyzed conversion of oil to biodiesel because they react with the catalyst to form soap, which deactivates the catalyst. ${ }^{3}$

Additional processing steps are required to use high FFA feedstock. The most common procedure, when FFA level is 5\% or less, is to add an extra amount of alkaline catalyst and allow its reaction with FFAs producing soap. The yield loss associated with this process will be at least twice the percentage of FFAs. ${ }^{3}$ Another strategy for feedstock containing 5-10\% of FFA is steam stripping. This process involves heating the oil to $290{ }^{\circ} \mathrm{C}$ under high vacuum and distilling FFAs from triglycerides. However, these two methods do not convert FFAs into biodiesel. Glycerolysis can also be used to

*e-mail: cah@uerj.br reduce FFA content. Free fatty acid and glycerol may react to produce glycerides and water. The water produced must be removed to favor the completion of the reaction, using high temperatures $\left(200-220^{\circ} \mathrm{C}\right)$ with an inert gas sparging or vacuum. The glycerides formed will also be transesterified during the alkaline-catalyzed process. Acid processing is the most common technique employed for feedstock with more than $10 \%$ of FFAs. An inorganic acid, such as sulfuric acid, is used as catalyst to convert FFAs into esters. Then, acid catalyst and the water, formed during the reaction, are removed before alkaline catalyst is added to promote transesterification reaction. ${ }^{3}$

An enzymatic process using lipases can overcome the drawbacks of the alkaline-catalyzed transesterification of acid oils. Lipases (triacylglycerol hydrolases, EC. 3.1.1.3) are hydrolases that catalyze in nature the hydrolysis of triglycerides into fatty acids and glycerol. However, in favorable thermodynamic conditions, they are also able to catalyze synthetic reactions like esterification, transesterification, acidolysis, alcoholysis, and aminolysis. The use of lipases allows simultaneous esterification of FFAs and transesterification of triglycerides present in the raw material forming alkyl esters. Besides, enzyme catalyzed reactions are highly selective, involve less energy consumption, require mild reaction conditions, provide an easier recovery of glycerol, and produce less waste., ${ }^{2,4}$

The reaction medium of biodiesel synthesis by transesterification of vegetable oils is a mixture of triglycerides, regioisomers of diglycerides or monoglycerides, and fatty acids. This mixture contains a wide range of substrates for enzyme action. ${ }^{5}$ Lipases obtained from different sources can exhibit different selectivity and activity in the presence of these very complex raw materials. Moreover, the support of the immobilized lipases could alter the biocatalyst performance in the presence of natural substrates like vegetable oils. ${ }^{5,6}$ The use of a mixture of lipases, which were obtained from different sources, have different specificities, and were immobilized on different supports, could enhance biodiesel rate and yield. There are several reports in which mixtures of different types of lipases were used successfully to catalyze transesterification and interesterification reactions. ${ }^{4,6-10}$ 
About $75 \%$ of the biodiesel produced in Brazil is derived from soybean oil. However, in Brazil, there are other sources of vegetable oils, like Jatropha curcas, Acrocomia aculeate, and palm oils that are interesting for biodiesel production, as they have higher productivity compared with soybean oil. On the other hand, these materials have the disadvantage of presenting high free fatty acid content. Thus, the development of technologies based on the use of acid oils for the production of biodiesel has been subject of studies aiming at reducing the biodiesel production costs.

The aim of this work was to study the production of biodiesel from acid soybean oil with ethanol in a batch system using lipase mixtures. As far as we are aware the use of combinations of lipases to catalyze biodiesel synthesis from acid soybean oil was not reported in the literature. The selected commercial lipases were Novozym 435, Lipozyme RM IM and Lipozyme TL IM (Novozymes). The effects of reaction temperature, enzyme concentration, and oil acidity in biodiesel synthesis with a single immobilized lipase were also investigated.

\section{EXPERIMENTAL}

\section{Material and methods}

\section{Reagents}

Refined soybean oil was provided by Sadia (Rio de Janeiro, Brazil) and presented an acid value (AV) of $0.5\left(\mathrm{mg}\right.$ of $\mathrm{KOH} \mathrm{g} \mathrm{g}^{-1}$ of oil). Model acid soybean oil was prepared by the addition of oleic acid to refined soybean oil in order to obtain oil samples with $\mathrm{AV}=8.5,50$ and 90. Ethanol (P.A.), butanol (P.A.), acetone (P.A.), sodium hydroxide (P.A.), n-hexane (99\%), and ethyl acetate (P.A.) were obtained from Vetec Química Fina Ltda (Rio de Janeiro, Brazil). Oleic acid (extra pure) and lauric acid were purchased from Merck (Darmstadt, Germany). Methyl heptadecanoate (99\%), monolaurin (99\%), dilaurin (99\%), trilaurin $(97 \%)$ were obtained from SigmaAldrich (St. Louis, USA).

\section{Commercial Lipases}

The commercial lipases used were Lipozyme RM IM (1,3-specific lipase from Rhizomucor miehei immobilized on an anionic resin), Lipozyme TL IM (1,3-specific lipase from Thermomyces lanuginosus immobilized on a gel of granulated silica), Novozym 435 (non-specific lipase from Candida antarctica immobilized on a macroporous acrylic resin). These biocatalysts were kindly donated by Novozymes Latin America Ltda (Brazil).

\section{Enzyme Activity Determination}

Esterification activity was determined by the consumption of oleic acid in the esterification reaction with butanol (oleic acid/ butanol molar ratio of 1) using $3 \mathrm{wt}$.\% of the biocatalyst at $45{ }^{\circ} \mathrm{C}$. The concentration of oleic acid in the samples was analyzed by acidbase volumetric analysis. ${ }^{11}$ One esterification unit (U) was defined as the enzyme amount that consumes $1 \mu \mathrm{mol}$ of oleic acid per minute under the experimental conditions. The esterification activities of commercial immobilized lipases Novozym 435, Lipozyme RM IM and Lipozyme TL IM were 3,876; 1,864 and $688 \mathrm{U} \mathrm{g}^{-1}$, respectively.

Transesterification activity was measured by the consumption of trilaurin in the transesterification reaction with ethanol (ethanol/ trilaurin molar ratio of 3) using $5 \mathrm{wt} . \%$ of the biocatalyst at $50{ }^{\circ} \mathrm{C}$. The concentration of trilaurin in the samples was analyzed by capillary gas chromatography according to the method described by Langone and Sant'Anna. ${ }^{12}$ One transesterification unit (U) was defined as the enzyme amount that consumes $1 \mu \mathrm{mol}$ of trilaurin per minute under the experimental conditions. The transesterification activities of commercial immobilized lipases Novozym 435, Lipozyme RM IM and Lipozyme TL IM were 3,202; 721 and 3,898 $\mathrm{U} \mathrm{g}^{-1}$, respectively.

\section{Biodiesel Synthesis}

The transesterification reactions between soybean oil and ethanol were carried out in a closed $15 \mathrm{~mL}$ batch reactor magnetically stirred, coupled to a condenser in order to avoid alcohol loss by volatilization. The reaction temperature was kept constant by circulating ethylene glycol from a thermostatic bath (Haake DC10) into the jacket of the reactor.

The mass of soybean oil used was $8 \mathrm{~g}$ in all experiments. This value corresponds to 0.01 moles of triglycerides and is based on the molar mass of oil, calculated from the oil composition $\left(801 \mathrm{~g} \mathrm{~mol}^{-1}\right)$. The alcohol addition was carried out in three stages at 0,30 and $60 \mathrm{~min}$ of reaction. The stepwise addition of ethanol was used to avoid lipase denaturation caused by a higher initial concentration of alcohol. The amount of fatty acid ethyl ester (FAEE) produced was determined in samples of $20 \mu \mathrm{L}$, taken in duplicate from the reaction medium, by gas chromatography.

\section{Effect of temperature}

The effect of temperature $\left(40,50,60,70\right.$ and $\left.78^{\circ} \mathrm{C}\right)$ was studied in the reactions carried out using stoichiometric molar ratio of ethanol/ oil (3/1) and 5 wt.\% (based on soybean oil weight) of biocatalyst (Novozym 435, Lipozyme RM IM and Lipozyme TL IM).

\section{Effect of enzyme concentration}

The enzyme concentration $(1,3,5,7$, and 9 wt.\% based on soybean oil weight) was investigated in the ethanolysis of soybean oil (ethanol/oil molar ratio of $3 / 1$ ) at $50{ }^{\circ} \mathrm{C}$.

\section{Effect of acid content}

The effect of acid content in soybean oil (acid value of $0.5,8.5$, 50 and 90) was investigated in the reactions carried out at $50{ }^{\circ} \mathrm{C}$ using stoichiometric alcohol amounts with respect to both reactants (ethanol/oil molar ratio $=3 / 1$ and ethanol/oleic acid $=1 / 1$ ), and $5 \mathrm{wt}$. $\%$ of commercial lipase. For Novozym 435, the reactions were also investigated at $70^{\circ} \mathrm{C}$. The consumption of oleic acid was monitored by acid-base volumetric analysis.

\section{Effect of the use of mixture of lipases}

Initially, the effect of the use of the mixture of immobilized commercial lipases was investigated in the reactions of transesterification of soybean oil with ethanol carried out at $50{ }^{\circ} \mathrm{C}$ using Lipozyme TL IM and Lipozyme RM IM added at different times (at the beginning or after $30 \mathrm{~min}$ ) and different amounts.

The biodiesel synthesis from acid oil was studied using $5 \mathrm{wt}$ \% of immobilized lipase (Novozym 435, Lipozyme RM IM and Lipozyme TL IM) at the beginning of the reaction and, after $240 \mathrm{~min}$, adding more lipase ( $2 \mathrm{wt} . \%)$ to the reaction medium. In these experiments, the total concentration of lipase was $7 \mathrm{wt} \%$ and the reaction was carried out for $5.5 \mathrm{~h}$.

\section{Ester content}

The fatty acid ethyl ester (FAEE) content in the reaction medium was determined by gas chromatography according to the method described by Corrêa et al. ${ }^{13}$ The samples were analyzed on a Varian gas chromatograph (CP-3380 model), equipped with a flame ionization detector (FID), a CP WAX 52 CB capillary column $30 \mathrm{~m} \times 0.25 \mathrm{~mm}$ $\mathrm{x} 0.25 \mathrm{~mm}$, and a split injection system with a $1 / 20$ ratio. Injector and detector temperatures were kept at 280 and $300^{\circ} \mathrm{C}$, respectively. The oven was initially maintained at $200{ }^{\circ} \mathrm{C}$ for $4.5 \mathrm{~min}$, then heated up to $250{ }^{\circ} \mathrm{C}$ at a $20^{\circ} \mathrm{C} \mathrm{min}^{-1}$ rate, and kept constant at the final temperature 
for $5 \mathrm{~min}$. Hydrogen was used as carrier gas at a $2 \mathrm{~mL} \mathrm{~min}^{-1}$ flow rate; column pressure was set at 20 psi. Methyl heptadecanoate was used as internal standard. The ester content was expressed as gram of ethyl esters produced per gram of sample.

\section{RESULTS AND DISCUSSION}

\section{Biodiesel synthesis using a single commercial immobilized lipase}

The enzymatic biodiesel synthesis using acid oil is affected by many factors such as temperature, enzyme concentration, and acid content. Therefore, initially, these factors were studied in the transesterification reaction of soybean oil with ethanol catalyzed by each of selected commercial immobilized lipases (Novozym 435, Lipozyme RM IM and Lipozyme TL IM). Ethanol was chosen to be the alcohol used in the reaction due to its large-scale production in Brazil, and to its production from renewable sources. Moreover, ethanol has a lower inhibitory effect on biocatalyst activity than methanol.

\section{Effect of temperature on biodiesel synthesis}

The biodiesel synthesis using refined soybean oil was investigated at $40,50,60,70$ and $78{ }^{\circ} \mathrm{C}$. The results are summarized in Table 1 .

Table 1. Ester content (wt.\%) after $240 \mathrm{~min}$ of transesterification reaction between refined soybean oil $(\mathrm{AV}=0.5)$ and ethanol

\begin{tabular}{lccccc}
\hline \multirow{2}{*}{ Lipase } & \multicolumn{5}{c}{ Temperature $\left({ }^{\circ} \mathrm{C}\right)$} \\
\cline { 2 - 6 } & 40 & 50 & 60 & 70 & 78 \\
\hline Novozym 435 & 34 & 35 & 40 & 60 & 39 \\
Lipozyme RM IM & 39 & 31 & 29 & 27 & 22 \\
Lipozyme TL IM & 50 & 52 & 40 & 30 & 30 \\
\hline
\end{tabular}

Reactions conditions: 5 wt.\% of commercial lipase, ethanol/soybean oil molar ratio of 3 , ethanol added in three steps (1/3 at $0 \mathrm{~min}, 1 / 3$ after $30 \mathrm{~min}$ and $1 / 3$ after $60 \mathrm{~min}$ ).

The fatty acid ethyl esters (FAEE) concentration increased with the increase of reaction temperature for the reactions catalyzed by Novozym 435 until $70{ }^{\circ} \mathrm{C}$. In contrast, when Lipozyme TL IM was used, the content of biodiesel decreased significantly with increasing temperature above $50^{\circ} \mathrm{C}$. With Lipozyme RM IM, a slightly decrease in ethyl ester content was observed at temperatures higher than $40^{\circ} \mathrm{C}$. The differences among these commercial lipases could be explained considering that the sensitivity of an enzyme to thermal denaturation can vary with different factors ( $\mathrm{pH}$, solvent, substrates, products, etc) and may differ from one protein to another. ${ }^{14}$

In order to avoid denaturation of the lipases Lipozyme RM IM and Lipozyme TL IM, the subsequent experiments were performed at $50{ }^{\circ} \mathrm{C}$.

\section{Influence of enzyme concentration}

The ester content using enzyme concentration from 1 to $9 \mathrm{wt} . \%$ after $240 \mathrm{~min}$ is presented in Table 2. The FAEE increased as the amount of lipase increased up to $7 \mathrm{wt} . \%$, above that the ethyl ester concentration remained almost constant for the reactions carried out with Lipozyme RM IM and TL IM. As reported in the literature, ${ }^{15,16}$ this effect can be explained by the formation of biocatalyst aggregates, which limit mass transfer processes.

However, in the reaction employing Novozym 435 the highest ester content $(73 \%)$ was reached with $9 \mathrm{wt} . \%$, and the initial rate of product formation was directly proportional to Novozym 435 concentration in the range studied (1 - $9 \mathrm{wt} . \%)$ (results not shown).
Although the best results were found for enzyme concentrations equal or higher than $7 \mathrm{wt} . \%$, the investigation of the influence of the experimental parameters (temperature, time course and acid value of the oil) was performed using $5 \mathrm{wt}$. $\%$ of biocatalyst to reach lower levels of ester contents (lower conversions) and thus let more evident the effect of each parameter.

Table 2. Ester content (wt.\%) after $240 \mathrm{~min}$ of transesterification reaction between refined soybean oil $(\mathrm{AV}=0.5)$ and ethanol at $50{ }^{\circ} \mathrm{C}$

\begin{tabular}{lccccc}
\hline \multirow{2}{*}{ Lipase } & \multicolumn{5}{c}{ Enzyme Content (wt. \%) } \\
\cline { 2 - 6 } & 1 & 3 & 5 & 7 & 9 \\
\hline Novozym 435 & 12.2 & 24.2 & 35 & 50 & 73 \\
Lipozyme RM IM & 3.4 & 21.5 & 32 & 50.7 & 51.1 \\
Lipozyme TL IM & 2 & 19 & 50 & 50 & 48 \\
\hline
\end{tabular}

Reactions conditions: ethanol/soybean oil molar ratio of 3 , ethanol added in three steps ( $1 / 3$ at $0 \mathrm{~min}, 1 / 3$ after $30 \mathrm{~min}$ and $1 / 3$ after $60 \mathrm{~min}$ ).

\section{Effect of oil acidity}

The influence of acid content in enzymatic biodiesel synthesis was studied at $50{ }^{\circ} \mathrm{C}$ using $5 \mathrm{wt} . \%$ of lipase (Novozym 435 , Lipozyme RM IM and Lipozyme TL IM). The results are presented in Table 3.

Table 3. Ester content (wt.\%) after $240 \mathrm{~min}$ of transesterification reaction between acid soybean oil and ethanol at $50{ }^{\circ} \mathrm{C}$

\begin{tabular}{lcccc}
\hline \multirow{2}{*}{ Lipase } & \multicolumn{4}{c}{ Acid Value } \\
\cline { 2 - 5 } & 0.5 & 8.5 & 50 & 90 \\
\hline Novozym 435 & 32 & 35 & 39 & 43 \\
Lipozyme RM IM & 31 & 32.2 & 52 & 46.9 \\
Lipozyme TL IM & 50 & 48 & 36 & 32 \\
\hline
\end{tabular}

Reactions conditions: 5 wt.\% of commercial lipase, ethanol/soybean oil molar ratio of 3 , ethanol added in three steps (1/3 at $0 \mathrm{~min}, 1 / 3$ after $30 \mathrm{~min}$ and $1 / 3$ after $60 \mathrm{~min}$ ).

As can be seen in Table 3, biodiesel was produced from the acid oils with all biocatalysts. Matassoli et al. ${ }^{17}$ studied biodiesel synthesis via alcoholysis of palm oil $(\mathrm{AV}=6.2)$ and verified only $3.5 \%$ of ester content using $1 \mathrm{wt} . \%$ of potassium hydroxide. Therefore, the results obtained by enzymatic synthesis of biodiesel from acid oils resulted in higher production of esters than observed for the alkalinecatalyzed process.

Lipozyme TL IM presents the highest ester content (close to 50\%) for soybean oils with AV up to 8.5, but for higher acid amounts the FAEE content decreased continuously. These results reflect the highest transesterification activity $\left(3,898 \mathrm{U} \mathrm{g}^{-1}\right)$ of this biocatalyst associated to its lowest esterification activity $\left(688 \mathrm{U} \mathrm{g}^{-1}\right)$.

Novozym 435 has high tolerance to oil acidity. A slight increase in ester content (from 32 to $43 \%$ ) was observed with the increase in oleic acid content in oil, which can be explained by its high esterification $\left(3,876 \mathrm{U} \mathrm{g}^{-1}\right)$ and transesterification $\left(3,202 \mathrm{U} \mathrm{g}^{-1}\right)$ activity. It is important to highlight that Novozym 435 is very stable in the presence of high content of low molecular weight alcohols, like ethanol. ${ }^{18,19}$ So, Novozym 435 can be a good biocatalyst for the biodiesel synthesis using soybean oil with high $\mathrm{AV}$, because this synthesis is carried out in the presence of a large excess of ethanol. ${ }^{20-22}$

The highest ester content (52\%) was verified in the reaction with soybean oil with acid value of 50 using Lipozyme RM IM. In fact, the literature reports that Lipozyme RM IM presents high esterification activity and high stability in anhydrous media. The FAEE content decreased when acid value of acid oil is 90 , probably due to the 
increase in water content due to the reaction of free fatty acid with ethanol. Water is produced during esterification reaction and must be removed to shift the chemical equilibrium toward products. Besides, high concentration of water in transesterification reaction catalyzed by Lipozyme RM IM could led to hydrolysis of the ester formed. ${ }^{23}$

The negative effect of water was verified by $\mathrm{Li}$ et al. ${ }^{9}$ in the methanolysis of waste and refined rapeseed oil. The final methyl ester $(\mathrm{ME})$ yield of waste oil $(\mathrm{AV}=135)$ was much lower than that of refined oil ( $\mathrm{AV}=3.5)$. The final ME yield obtained in the presence of molecular sieves into the reaction medium of waste oil was as high as that obtained with refined oil.

Since Novozym 435 showed higher catalytic performance at $70{ }^{\circ} \mathrm{C}$ (Table 1), experiments were also performed at this temperature with acid soybean oil and the results are presented in Table 4 . The ester content decreased with the increase of acid value in the reactions carried out at $70{ }^{\circ} \mathrm{C}: 44 \%, 41 \%$ and $28 \%$ for the oils with acid values of $8.5,50$, and 90 , respectively. Probably, the increase of temperature associated with the increase of ethanol (hydrophilic reagent) amount in the reaction medium, due to high acid content, resulted in a decrease in enzyme stability. According to these results, the use of mixtures of commercial immobilized lipases for biodiesel synthesis from acid oils was studied at $50{ }^{\circ} \mathrm{C}$.

Table 4. Ester content (wt. \%) after $240 \mathrm{~min}$ of transesterification reaction between acid soybean oil and ethanol at $70{ }^{\circ} \mathrm{C}$

\begin{tabular}{lcccc}
\hline \multirow{2}{*}{ Biocatalyst } & \multicolumn{5}{c}{ Acid Value } \\
\cline { 2 - 5 } & 0.5 & 8.5 & 50 & 90 \\
\hline Novozym 435 & 60 & 44 & 41 & 28
\end{tabular}

Reactions conditions: 5 wt.\% of commercial lipase, ethanol/soybean oil molar ratio of 3 , ethanol added in three steps $(1 / 3$ at $0 \mathrm{~min}, 1 / 3$ after $30 \mathrm{~min}$ and $1 / 3$ after $60 \mathrm{~min}$ ).

The acid values were also determined in the enzymatic synthesis of biodiesel from acid oil. It can be seen in Figure 1a that Novozym 435 converted almost all FFA into ethyl ester after $240 \mathrm{~min}$. A similar profile was observed in the reactions catalyzed by Lipozyme RM IM using soybean oil with acid value of 8.5 and 50 (Figure 1b). A conversion higher than $75 \%$ was obtained using oil with AV $=90$ and 5 wt.\% of Lipozyme RM IM. As expected, considering the low esterification activity of Lipozyme TL IM, the acid value did not very significantly for all tested acid oil (Figure 1c).

\section{Biodiesel Synthesis Using Mixtures of Commercial Immobilized Lipases}

A typical soybean oil is composed of $2.3-11 \%$ palmitic acid, $2.4-6 \%$ stearic acid, $23.5-31 \%$ oleic acid, $49-51.5 \%$ linoleic acid, $2-10.5 \%$ linolenic acid. ${ }^{24}$ This diversity of alkyl fatty acids could affect enzyme activity. The lipases obtained from different microorganisms can exhibit different selectivities and activities, so the combined use of two immobilized lipases in the transesterification reaction of soybean oil was investigated trying to decrease catalyst cost and increase biodiesel yield.

Initially, to explore the possibility of synergistic effect, Lipozyme RM IM and Lipozyme TL IM were mixed in low concentrations and in different reaction times. These lipases are less expensive than Novozym 435, and their combination could represent a more efficient and lower cost biocatalyst. The use of smaller amount of biocatalyst (3 wt.\%) in these experiments aims to prevent the leveling off effect that could be observed when higher conversions were obtained. The time course for transesterification of soybean oil catalyzed by combined or pure lipases is presented in Figure 2.
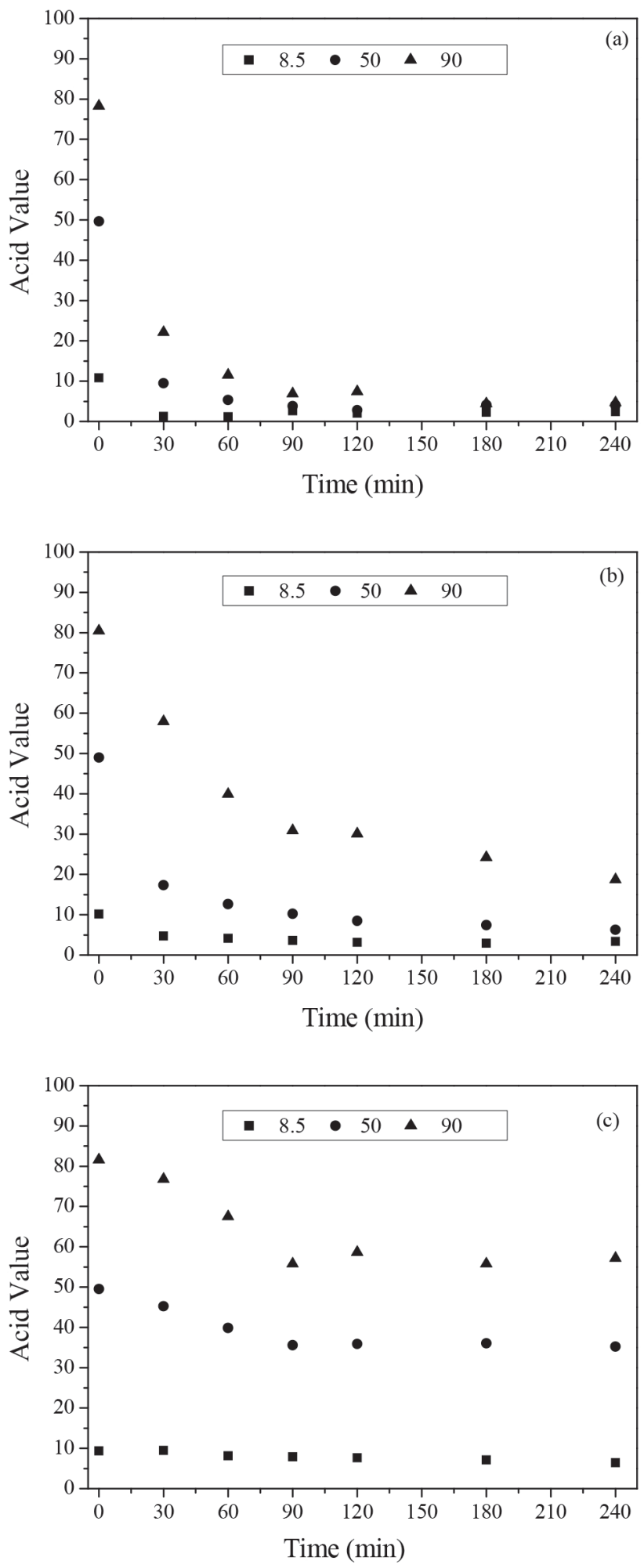

Figure 1. Acid value (mg of $\mathrm{KOH} \mathrm{g}^{-1}$ of oil) in the transesterification reaction using acid soybean oil ( $A V$ of 8.5, 50 and 90) during 240 min. Reaction conditions: 5 wt. \% of lipase, ethanol/soybean oil molar ratio of 3 , oleic acid/ ethanol molar ratio of 1 , ethanol added in three steps (1/3 at $0 \mathrm{~min}, 1 / 3$ after $30 \mathrm{~min}$ and $1 / 3$ after $60 \mathrm{~min}$ ), at $50^{\circ} \mathrm{C}$. (a) Novozym 435; (b) Lipozyme RM IM; (c) Lipozyme TL IM

The enzymes were added at the beginning and/or after $30 \mathrm{~min}$ of reaction, in a concentration of $1.5 \mathrm{wt} . \%$. Control experiments using 3 wt.\% of lipase (Lipozyme TL IM or Lipozyme RM IM) were 


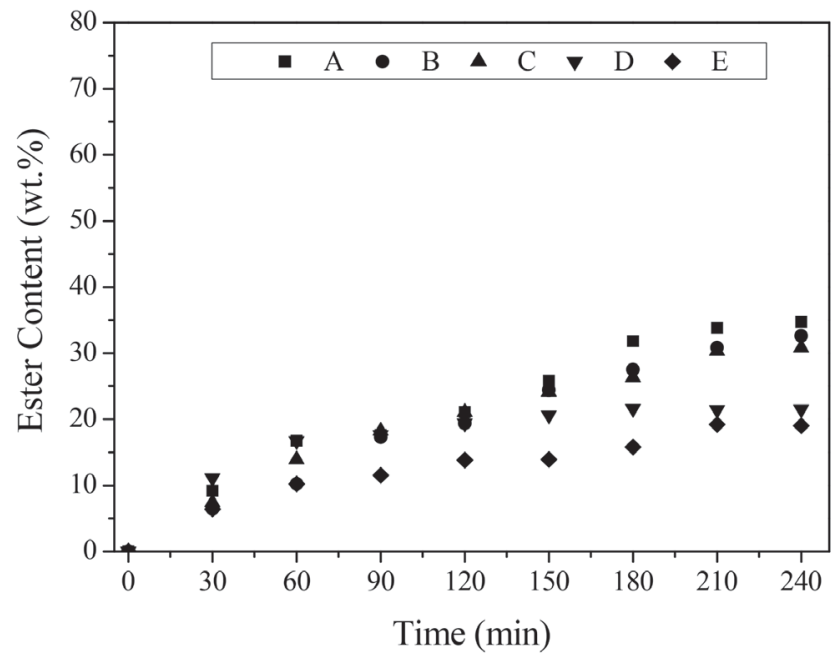

Figure 2. Ester content (wt.\%) in the transesterification reaction between soybean oil and ethanol using ethanol/soybean oil molar ratio of 3, ethanol added in three steps (1/3 at $0 \mathrm{~min}, 1 / 3$ after $30 \mathrm{~min}$ and $1 / 3$ after $60 \mathrm{~min}$ ) at $50{ }^{\circ} \mathrm{C}$ (A) $1.5 \mathrm{wt}$ \% Lipozyme RM IM and $1.5 \mathrm{wt}$. \% Lipozyme TL IM at $t=0$ min; (B) 1.5 wt.\% Lipozyme RM IM at $t=0$ min and $1.5 \mathrm{wt} . \%$ Lipozyme TL $I M$ at $t=30 \mathrm{~min} ;(C) 1.5 \mathrm{wt} . \%$ Lipozyme TL IM at $t=0 \mathrm{~min} \mathrm{t}$ and $1.5 \mathrm{wt} . \%$ Lipozyme RM IM at $t=30 \mathrm{~min}$; (D) $3.0 \mathrm{wt}$.\% Lipozyme TL IM at $t=0 \mathrm{~min}$; (E) 3.0 wt. \% Lipozyme RM IM at $t=0 \mathrm{~min}$

carried out to evaluate the influence of the mixture of Lipozyme TL IM (1.5 wt.\%) and Lipozyme RM IM (1.5 wt.\%) in the amount of ester produced. Clearly, the blend of lipases gave the best results. All the experiments carried out with combined use of lipases resulted in an increase in ethyl esters content mainly after $180 \mathrm{~min}$ of reaction, but the addition of enzyme preparations at different reaction times (at 0 or $30 \mathrm{~min}$ ) and the order of lipase addition did not result in a significant difference in ester content after $240 \mathrm{~min}$. These enzymes are recognized as 1,3-specific lipases, but their fatty acid specificities are not equal ${ }^{5}$ and could justify the increase in the ester content. The mixture of Lipozyme TL IM and Lipozyme RM IM, added in the beginning of the reaction (Figure 2A), resulted in an increase of $75 \%$ in the ester content after $240 \mathrm{~min}$, when compared with the amount obtained with 3 wt.\% of Lipozyme TL IM.

Rodrigues and Ayub ${ }^{5}$ observed that the blend of Lipozyme RM IM and Lipozyme TL IM gave best results for transesterification of soybean oil, producing near $10 \%$ more biodiesel than Lipozyme TL IM and 50\% more than Lipozyme RM IM. Ibrahim et al. ${ }^{6}$ also observed a synergistic effect for the Lipozyme TL IM - RM IM dual system in the interesterification of palm stearin and coconut oil.

Another experiment was performed using different amounts of Lipozyme TL IM (2, 4 and 6 wt.\%) and the same content of Lipozyme RM IM (1 wt.\%), in comparison with single lipase-catalyzed reactions $(3,5$, and 7 wt.\%). The results in Figure 3 indicated a positive synergistic effect for lipase combinations, which depends on the amount of lipase. The use of combined enzyme resulted in higher amount of ester produced when the total amount of lipases was lower than $7 \mathrm{wt} . \%$. Ibrahim et al. ${ }^{6}$ demonstrated that a positive interaction between two mixed lipases does not always happen. According to these authors, the interactive effect differs for different lipase combinations and mixing ratios, and some experiments with blends of different lipases showed an antergic effect. ${ }^{6}$

Considering these results, the enzymatic synthesis of biodiesel from acid oil using a mixture of lipases in a total concentration of $7 \mathrm{wt} . \%$ was further investigated. According to the results shown in Table 2 and Figure 3, an amount of Lipozyme TL IM and RM IM higher than $7 \mathrm{wt} . \%$ did not result in higher ester content.

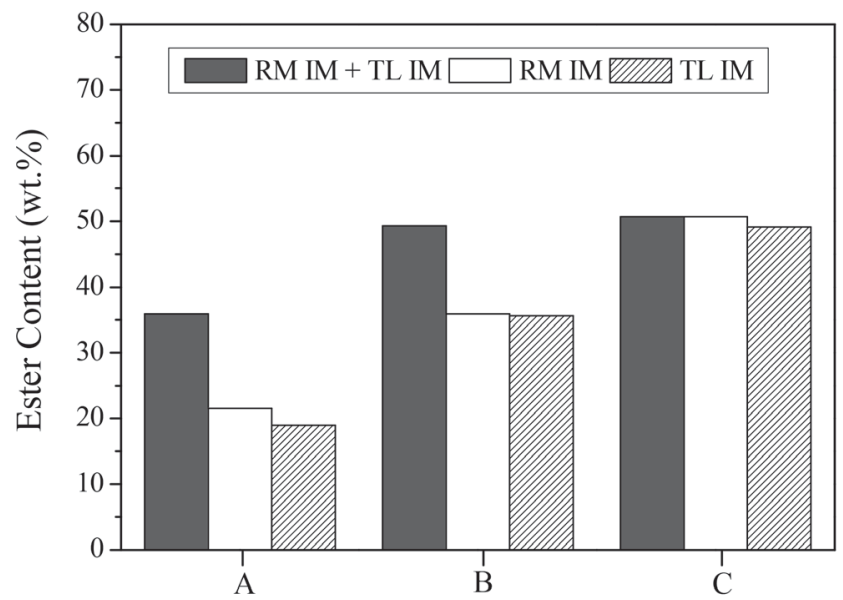

Figure 3. Ester content (wt.\%) after 4 h of the transesterification reaction between soybean oil and ethanol using ethanol/soybean oil molar ratio of 3 , ethanol added in three steps $(1 / 3$ at $0 \mathrm{~h}, 1 / 3$ after $0.5 \mathrm{~h}$ and $1 / 3$ after $1 \mathrm{~h})$ at $50{ }^{\circ} \mathrm{C}$ (A) 3 wt.\% lipase: 3 wt.\% Lipozyme RL IM, 3 wt.\% Lipozyme TL IM, and mixture with 1 wt.\% Lipozyme RM IM and 2 wt.\% Lipozyme TL IM (B) 5 wt.\% lipase: 5 wt.\% Lipozyme RL IM, 5 wt.\% Lipozyme TL IM, and mixture with 1 wt.\% Lipozyme RM IM and 4 wt.\% Lipozyme TL IM (C) 7 wt. \% lipase: 7 wt. \% Lipozyme RL IM, 7 wt.\% Lipozyme TL IM, and mixture with 1 wt.\% Lipozyme RM IM and 6 wt.\% Lipozyme TL IM

So, the experiments with acid oil were carried out adding $5 \mathrm{wt} . \%$ of the first lipase at the beginning of the reaction. The second lipase was added after $240 \mathrm{~min}$. A control experiment was made adding more $2 \mathrm{wt} . \%$ of the initial lipase after 240 min considering that inactivation of the enzyme can occur along the reaction and that the increase in the amount of catalyst could promote an increase in the production of ethyl esters. The time course of enzymatic transesterification of acid oil with lipase mixture was shown in Figures 4-6.

It can be noticed that enzyme addition after $240 \mathrm{~min}$ increased FAEE synthesis in all experiments. Moreover, this effect is more significant for the mixture of lipases. The maximum synergistic effect was achieved with combined use of Lipozyme TL IM and Novozym 435 in transesterification reaction of acid soybean oil with $\mathrm{AV}=50$ and 90 (Figures 5c, 6a, 6c). A significant increase in the ester content, after $330 \mathrm{~min}$, was observed in those reactions.

As observed by several authors, ${ }^{4-9}$ a mixture of two or more lipases can contribute to increase the ester yield in biodiesel production. This increase can be explained by the different specificities of each lipase and different substrates present in soybean oil. The highest theoretical yield using lipase with 1,3-positional specificity should be $67 \%$. Yield higher than $67 \%$ may be due to acyl migration from the $s n-2$ position to the $s n-1,3$ position that occurs during the reaction. The use of a non-specific lipase can catalyze the transesterification of 2-monoglyceride to give fatty acid ethyl ester and glycerol.

Huang et al. ${ }^{4}$ observed that during the first $0.5 \mathrm{~h}$, the reaction rate of the transesterification reaction of lard oil with methanol catalyzed by combined lipases (Lipozyme TL IM + Novozym 435) was the fastest, followed by those of the Lipozyme TL IM and Novozym 435. According to the authors, this phenomenon might be due to the fact that joining a non-specific lipase (Novozym 435) and an 1,3-specific lipase (Lipozyme TL IM) could accelerate the transesterification reaction rate at 1 - and 3 -sites and meanwhile eliminate the limitation of the acyl migration at 2-site, which is considered to be the confined step of transesterification reaction.

In the experiments carried out with acid oil of $\mathrm{AV}=8.5$ (Figures 4 a,b,c), the highest reaction rate was obtained with Lipozyme TL IM. The ester content was about 30\%, after $60 \mathrm{~min}$, 

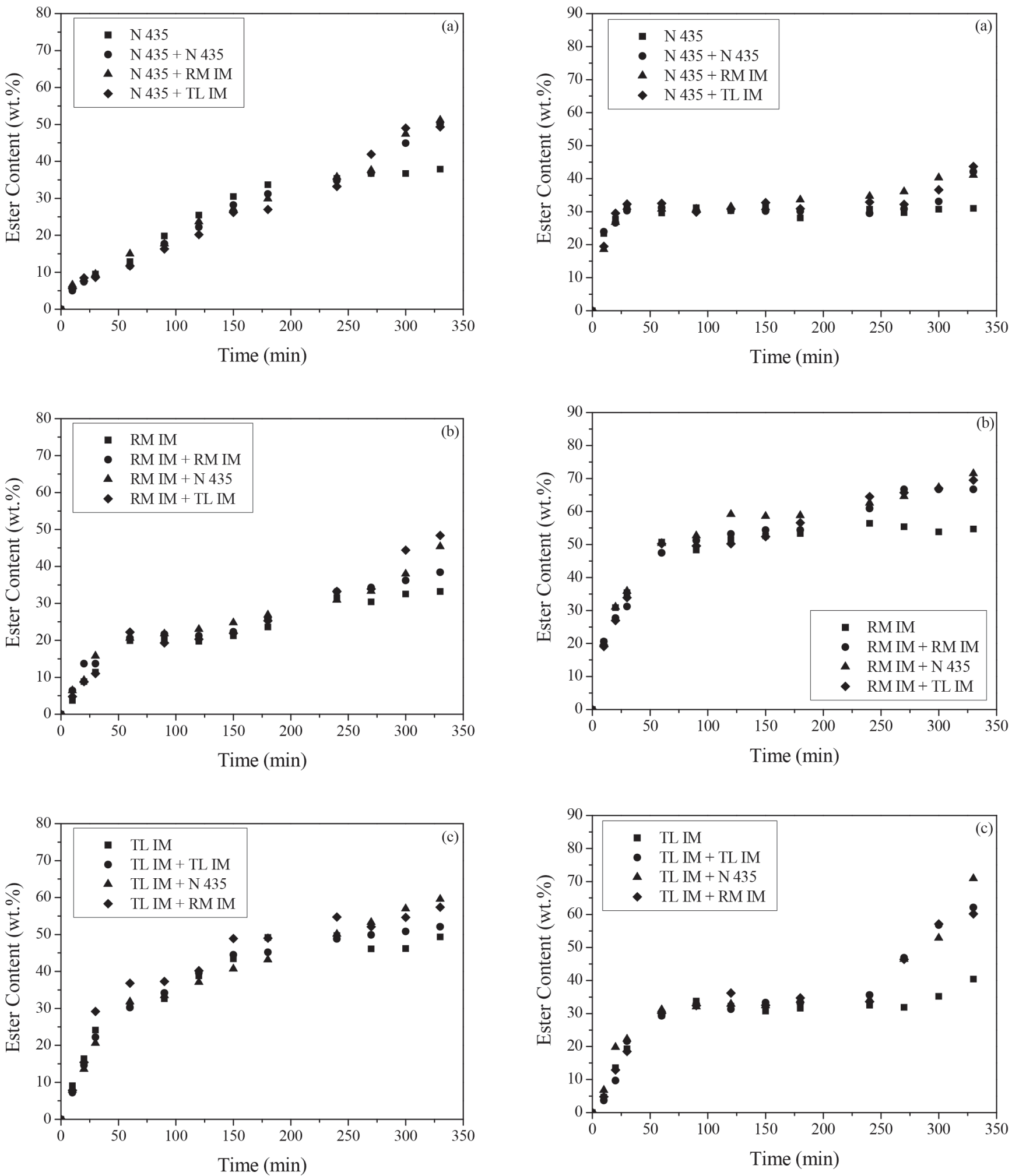

Figure 4. Ester content (wt. \%) in the transesterification reaction between acid soybean oil $(A V=8.5)$ and ethanol at $50{ }^{\circ} \mathrm{C}$. Reaction conditions: mixture of commercial lipases (5 wt.\% of biocatalyst 1 added at time zero and $2 \mathrm{wt} . \%$ of biocatalyst 2 added after $4 \mathrm{~h}$ of reaction), ethanol/soybean oil molar ratio of 3 , oleic acid/ethanol molar ratio of 1 , ethanol added in three steps (1/3 at 0 h, $1 / 3$ after $0.5 \mathrm{~h}$ and $1 / 3$ after 1 h). Biocatalyst $1:$ (a) Novozym 435, (b) Lipozyme RM IM, (c) Lipozyme TL IM

using Lipozyme TL IM whereas for Novozym 435 and Lipozyme RM IM it was $13 \%$ and $20 \%$, respectively. However, increasing acid

Figure 5. Ester content (wt.\%) in the transesterification reaction between acid soybean oil $(A V=50)$ and ethanol at $50{ }^{\circ} \mathrm{C}$. Reaction conditions: mixture of commercial lipases (5 wt.\% of biocatalyst 1 added at time zero and $2 \mathrm{wt} . \%$ of biocatalyst 2 added after 4 h of reaction), ethanol/soybean oil molar ratio of 3, oleic acid/ethanol molar ratio of 1, ethanol added in three steps (1/3 at $0 \mathrm{~h}, 1 / 3$ after $0.5 \mathrm{~h}$ and $1 / 3$ after $1 \mathrm{~h}$ ). Biocatalyst 1 : (a) Novozym 435, (b) Lipozyme RM IM, (c) Lipozyme TL IM

content (Figures 5c, 6c) FAEE content decreased. It can be predicted considering that Lipozyme TL IM has the lowest esterification activity 

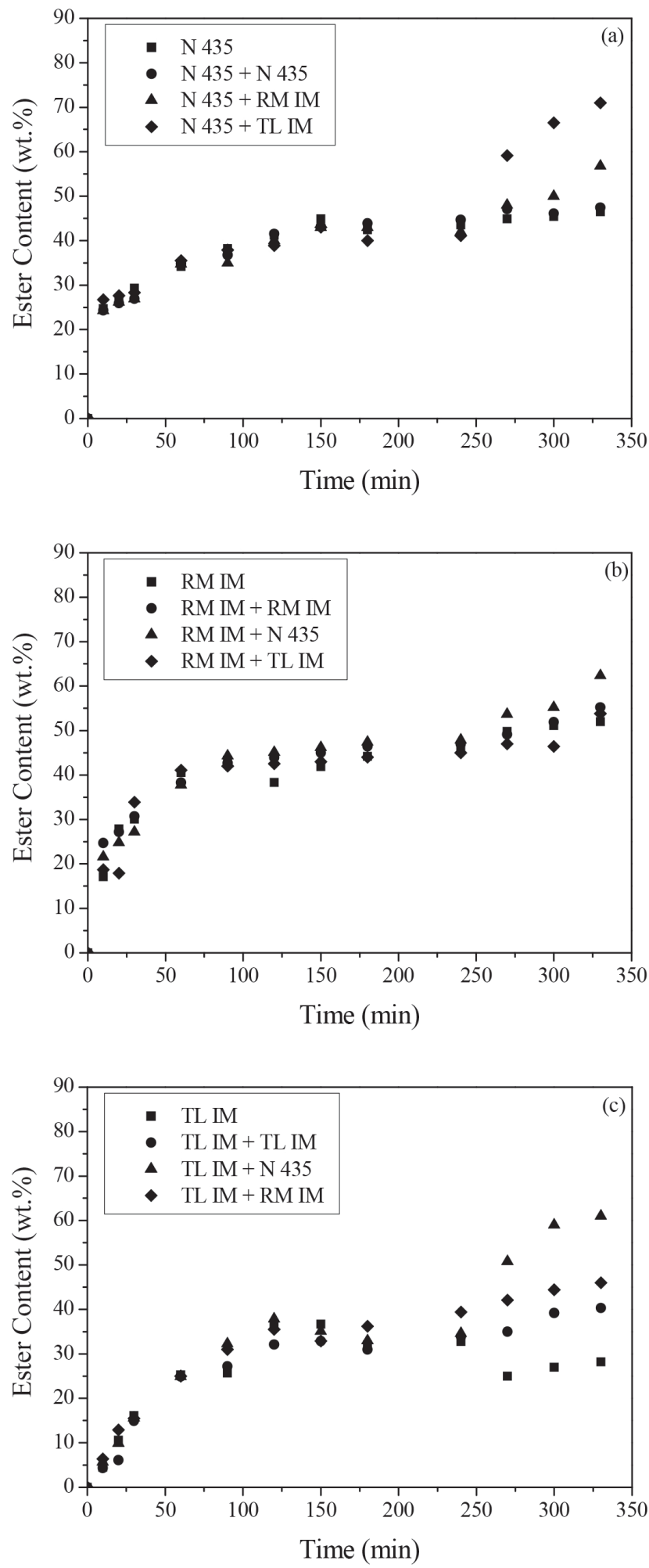

Figure 6. Ester content (wt.\%) in the transesterification reaction between acid soybean oil $(A V=90)$ and ethanol at $50{ }^{\circ} \mathrm{C}$. Reaction conditions: mixture of commercial lipases (5 wt.\% of biocatalyst 1 added at time zero and $2 \mathrm{wt} . \%$ of biocatalyst 2 added after 4 h of reaction), ethanol/soybean oil molar ratio of 3 , oleic acid/ethanol molar ratio of 1 , ethanol added in three steps (1/3 at 0 h, 1/3 after $0.5 \mathrm{~h}$ and $1 / 3$ after 1 h). Biocatalyst 1: (a) Novozym 435, (b) Lipozyme RM IM, (c) Lipozyme TL IM

compared with Novozym 435 and Lipozyme RM IM. Therefore, the addition of fresh lipase with high esterification activity, as
Lipozyme RM IM and Novozym 435, after 240 min, enhanced biodiesel synthesis. Moreover, the lower ester contents obtained in the reactions carried out with acid oil with $\mathrm{AV}=90$ can be related to the negative effect of the presence of the water produced by FFAs esterification. It is well known that water activity of the reaction medium is an important parameter which influences reaction rates/ yields in enzyme catalyzed reactions in low water media. ${ }^{25}$ Besides, the higher ethanol amount used in these experiments could also cause enzymatic inactivation.

In the experiments shown in Figures 4-6, we can also see that esterification reactions are faster than transesterification ones. ${ }^{8}$ The initial rates observed in the reactions with high acid content (AV $=50$ and 90) were higher than those obtained with $\mathrm{AV}=8.5$ using Lipozyme RM IM and Novozym 435 (Figures 4a,b) and Figures 5a,b). Li et al. ${ }^{9}$ also observed that the initial reaction rate of methanolysis of waste oil (AV = 135) catalyzed by mix of Lipozyme TL IM (3\%) with Novozym $435(1 \%)$ was faster than that of the methanolysis of refined oil $(\mathrm{AV}=3.5)$ due to the high FFAs content.

\section{CONCLUSION}

Immobilized lipases Lipozyme RM IM, Lipozyme TL IM and Novozym 435 were used to catalyze biodiesel synthesis from soybean oil with different levels of acidity ( $\mathrm{AV}=0.5,8.5,50$ and 90). All tested biocatalysts were active for biodiesel production and ester contents close to $50 \%$ were obtained even without optimizing the experimental conditions. High fatty acid conversion was obtained with Novozym 435 and Lipozyme RM IM. Therefore, commercial immobilized lipases can be used to produce biodiesel from high levels acidity oils that represent a raw material cheaper than refined vegetable oils. This process has potential to be used as a pre-step to alkaline-catalyzed transesterification. The use of mixtures of commercial immobilized lipases was proposed in order to guarantee an effective and cost-efficient biodiesel production from acid oils. The blend of Lipozyme TL IM and Novozym 435 is the best option taking into account biocatalyst cost and biodiesel yield. The main reason is the low cost of Lipozyme TL IM that is less expensive than Novozym 435.

\section{ACKNOWLEDGEMENTS}

The authors would like to thank to CNPq for financial support.

\section{REFERENCES}

1. Jegannathan, K. R.; Wang Ting, V. F.; Pogaku, R.; Biomass Bioenergy 2011, 35, 4221.

2. Aarthy, M.; Saravanan, P.; Gowthaman, M. K.; Rose, C.; Kamini, N. R.; Chem. Eng. Res. Des. 2014, 92, 1591.

3. Shrestha, D. S.; Van Gerpen, J. In Industrial Crops and Uses; Singh, B. P., ed.; CABI: Oxfordshire, 2010.

4. Huang, Y.; Zheng, H.; Yan, Y.; Appl. Biochem. Biotechnol. 2010, 160, 504.

5. Rodrigues, R. C.; Ayub, M. A. Z.; Process Biochem. 2011, 46, 682.

6. Ibrahim, N. A.; Guo, Z.; Xu, X.; J. Am. Oil. Chem. Soc. 2008, 85, 37.

7. Lee, D. H.; Kim, J. M.; Shin H. Y.; Kang, S. W.; Kim, S. W.; Biotechnol. Bioprocess Eng. 2006, 11,522.

8. Guan, F.; Peng, P.; Wang, G.; Yin, T.; Peng, Q.; Huang, J.; Guan, G.; Li, Y.; Process. Biochem. 2010, 45, 1677.

9. Li, L.; Du, W.; Liu, D.; Wang, L.; Li, Z.; J. Mol. Catal. B: Enzym. 2006, 43,58 .

10. Banerjee, A.; Singh, V.; Solanki, K.; Mukherjee, J.; Gupta, M. N.; Sustain. Chem. Processes 2013, $1,1$. 
11. Souza, M. S.; Aguieiras, E. C. G.; Silva, M. A. P.; Langone, M. A. P.; Appl. Biochem. Biotechnol. 2009, 154, 253.

12. Langone, M. A. P.; Sant'Anna, G. L. Jr.; Appl. Biochem. Biotechnol. 1999, 77, 759 .

13. Corrêa, I. N. S.; Souza, S. L.; Catran, M.; Bernardes, O. L.; Portilho, M. F.; Langone, M. A. P.; Enzyme Res. 2011, 1.

14. Bulcholz, K.; Kasche, V.; Bornscheuer, U. T.; Biocatalysis and Enzyme Technology; Wiley-VCH: Weinheim, 2005.

15. Aguieiras, E. C. G.; Veloso, C. O.; Bevilaqua, J. V.; Rosas, D. O.; Silva, M. A. P.; Langone, M. A. P.; Enzyme Res. 2011, 1.

16. Cerveró, J. M.; Álvarez, J. R.; Luque, S.; Biomass Bioenergy 2014, 61, 131.

17. Matassoli, A. L. F.; Corrêa, I. N. S.; Portilho, M. F.; Veloso, C. O; Langone, M. A. P.; Appl. Biochem. Biotechnol. 2009, 155, 44.
18. Rosset, I. G.; Tavares, M. C. H.; Assaf, E. M.; Porto, A. L. M.; Appl. Catal., A 2011, 392, 136.

19. Rosset, I. G.; Tavares, M. C. H.; Assaf, E. M.; Porto, A. L. M.; Catal. Lett. 2013, 143, 863.

20. Talukder, M. M. R.; Wu, J. C.; Lau, S. K.; Cui, L.C.; Shimin, G.; Lim, A.; Energy Fuels 2009, 23, 1.

21. Hernández-Martín, E.; Otero, C.; Bioresour. Technol. 2008, 99, 277.

22. Torres, C. F.; Hill, C. G.; Otero. C.; Biotechnol. Prog. 2004, $20,756$.

23. Rodrigues R. C.; Fernandez-Lafuente, R.; J. Mol. Catal. B: Enzym. 2010, 66, 15 .

24. Rinaldi, R.; Garcia, C.; Marciniuk, L. L.; Rossi, A. V.; Schuchardt, U.; Quim. Nova 2007, 30, 1374.

25. Adlercreutz, P. In Organic Synthesis with Enzymes in Non-Aqueous Media; Carrea, G., Riva, S., eds.; Wiley-VCH: Weinheim, 2008. 